\title{
BIRD DIVERSITY, COMPOSITION AND RESPONSE DURING COVID-19 IN AN URBAN LANDSCAPE, JAMALPUR, BANGLADESH
}

\author{
Ashikur Rahman Shome, M. Firoj Jaman*, Md. Fazle Rabbe \\ and Md. Mahabub Alam \\ Department of Zoology, University of Dhaka, Dhaka-1000, Bangladesh
}

Keywords: Bird diversity, Species, COVID 19, Urban landscape, Jamalpur

\begin{abstract}
The world has been encountering a COVID-19 pandemic since late 2019. The world's people have also witnessed the free movement of wildlife, even in many urban areas in some countries during the imposed lockdown. We conducted research on the birds in the urban landscape of Jamalpur Sadar upazila from March 2020 to October 2020 during this pandemic situation. A total of 134 species of birds with 4338 individuals were recorded during the study period. The number of resident bird species was the maximum (115 species, $85.82 \%$ ) compared to migrants. The highest number of birds was observed (120 species, $89.55 \%$ ) and counted 2278 individual birds $52.51 \%$ ) in the summer season. It was observed that the highest diversity of birds ( 89 species, 65.92\%) was in March. The tree was the most ( 94 species, $\mathrm{n}=2502$ ) used microhabitat by birds in the study area. Among all birds, Pycnonotus cafer was the most abundant bird species with the highest relative abundance $(6.11 \%)$. Some bird species (e.g. Metopidius indicus, Anastomus oscitans, Amaurornis phoenicurus, Streptopelia tranquebarica, Lonchura malacca) were frequently observed in the urban landscape of the study area during the pandemic. Proper management and awareness creation are essential for the conservation of the bird species in this area.
\end{abstract}

\section{Introduction}

Globally the number of the human population is increasing very rapidly, which provides velocity to urbanization. This urbanization process is slowly changing the structure of landscape ecology. As a result, biodiversity is facing different anthropogenic threats ${ }^{(1,2)}$. Birds are one of the most common wild fauna in the urban area and due to the rapid urbanization-expansion process, it's population is facing threats and has already been declining ${ }^{(3-6)}$. Some studies suggested that urban habitats offer many advantages to wild animals, such as food accessibility, lower predation rate, etc., thus supporting more species relative to other habitats ${ }^{(7-10)}$. Situations in urban landscapes like less human disturbance and suitable space for wild animals can gather more species increasing the probability of detection ${ }^{(11)}$. During the COVID-19 situation, bird diversity and population seems to be increased in the study area.

The COVID-19 created an unexpected pandemic situation worldwide. To inhibit the spreading of the virus, most countries implemented different methods like social distancing, lockdown, and home-office ${ }^{(12)}$. In that snappy situation, a dramatic effect on the environment was

\footnotetext{
*Author for correspondence: <mfjaman4@gmail.com>.
} 
observed throughout the world by marked reduction of air ${ }^{(13)}$ and noise pollution ${ }^{(14)}$. The streets and roads of the urban areas became empty, movements of humans were small, which provide the spaces for the wildlife's unrestricted movement. This resulted in the observation of the unusual movement of wild animals ${ }^{(10)}$. Humans understood how wildlife is affected by human activities in urban areas, which was mentioned in social, print, and electronic media ${ }^{(11,12)}$.

Bangladesh is a small south-Asian country with diverse wildlife assets due to its geographical location ${ }^{(15-17)}$. The bird is a very familiar group of wildlife in Bangladesh that provides ecological services in natural and modified ecosystems. Globally, there are 9,026 living species of birds, and of them, $7.64 \%$ of species are found in Bangladesh ${ }^{(17)}$. Avifaunal diversity is one of the most important ecological indicators for estimating the health and quality of ecosystems. Apart from being a part of the food web, birds play vital roles in many crucial natural activities like pollination, seed dispersal, and pest control ${ }^{(18)}$. They also meet copious threats, remarkably anthropogenic activities that include indiscriminate killing, hunting, poisoning and trapping, habitat loss and its subsequent effect on food, and shelter; thus compelling birds to change their habitats ${ }^{(15,16,19)}$. Comprehensive and baseline studies are imperative for monitoring and preserving them from critical conditions ${ }^{(19,20)}$. Besides, it is important to observe birds' composition and diversity in the urban areas of Bangladesh in this pandemic situation methodologically so that it may allow comparing this data with the previously collected data during the normal situation.

We investigated the diversity and composition of birds to the sudden and drastic changes occurring in urban environments resulting from the COVID-19 lockdown in urban sites of the northern Jamalpur district of Bangladesh. No previous studies on birds are available in this study area. This study provides baseline information on diversity, status, abundance, seasonal occurrence, and habitat usage of birds in the pandemic situation that may help compare the data while monitoring birds in the future study area. This information on birds may also help for the conservation and management of birds.

\section{Materials and Methods}

Study area: Jamalpur Sadar upazila (24⒌5 $\left.55.4^{\prime \prime N} 89^{\circ} 56^{\prime} 37.7^{\prime \prime E}\right)$ is situated in the northern region of Bangladesh (Fig. 1). This study was conducted in the urban landscape $\left(55.25 \mathrm{Km}^{2}\right)$ of Jamalpur Sadar upazila in ten sites. The study sites have buildings, roads, urban settlements, homestead forests including different native and planted trees, grasslands, agricultural lands, fallow land, canal, river, permanent and temporary water body, ditch, ponds.

Data collection: We collected data through direct field observations from March 2020 to October 2020. The study period falls into two seasons: summer (March-June) and rainy (JulyOctober). We spent 32 days ( 4 days $\times 8$ months) in total at the field and observation was done in the early morning $(6.30 \mathrm{am}-10.00 \mathrm{am})$ and afternoon $(04.00 \mathrm{pm}-06.00 \mathrm{pm})$. We employed the transect method $^{(21)}$ for data collection. Sometimes opportunistic surveys were done during the study period. The size of the transect line was $500 \times 200$ square meters. In total, 10 fixed transects were selected and each transect was surveyed at least 2 times in each season. The habitats were 
categorized into 3 macrohabitats (arboreal such as trees, terrestrial such as grounds, and aquatic such as canal, ponds, etc.) and 10 microhabitats are bushy area (enrich with herbs), fallow land (lands without trees or infrastructure), roadside (the area around the road), mudflat (area shallow waterbody), grassland (up to one meter), tree, urban settlement (poles, buildings, towers, etc.), permanent waterbody (water present throughout the year, e.g., river, canal, pond, etc.), temporary waterbody (water found only during the rainy season) and agricultural land (cultivated land, farmland). Microhabitats are classified according to identifying characteristics of each habitat in the study area under 3 basic macro habitats.

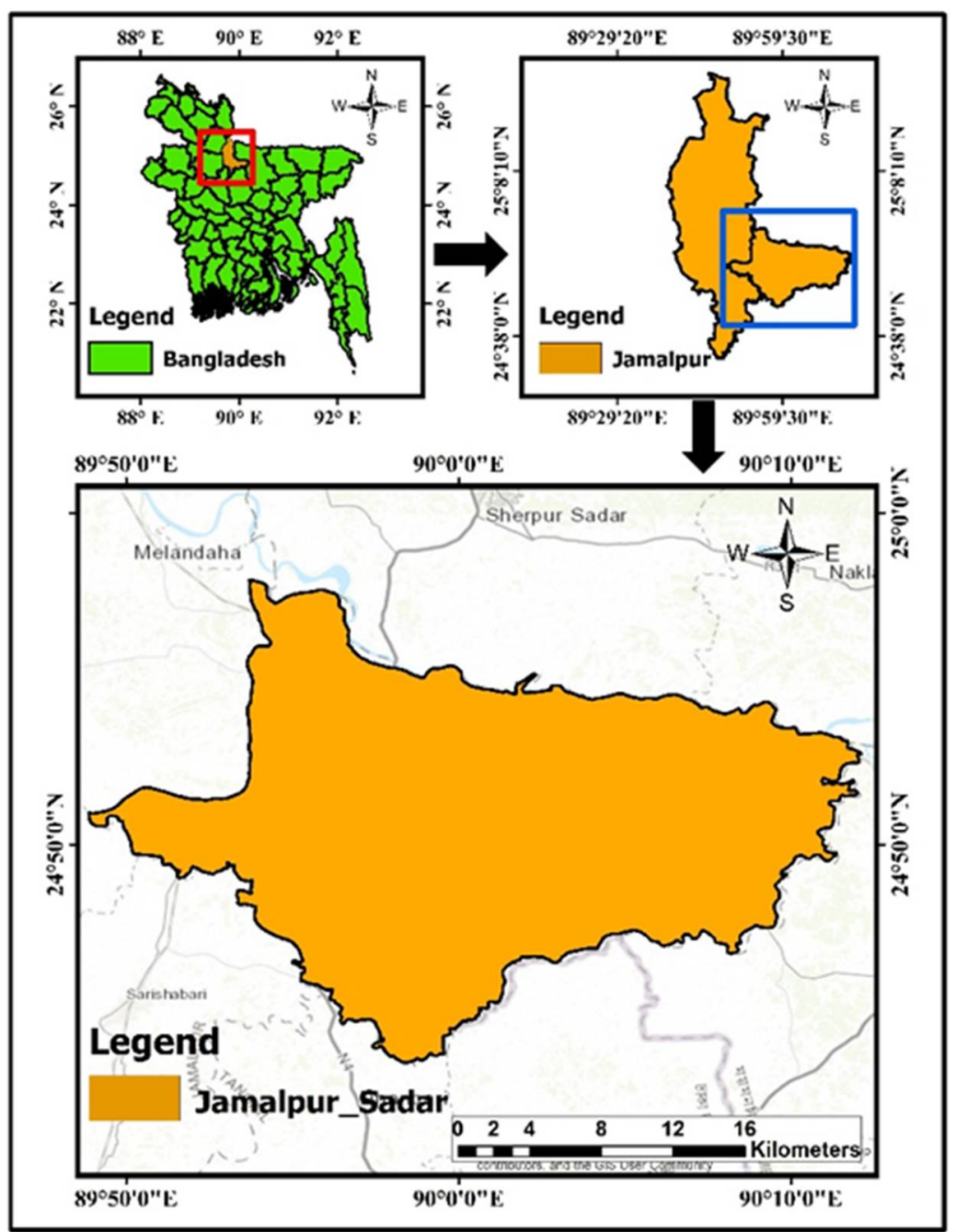

Fig. 1. Map of the Jamalpur Sadar upazila 
Some species of birds were recorded by receiving their song and call sound, which were normally hidden in the bushes, jungles, and branches of trees. The calls were recorded by the Samsung A50 Phone, which the authors later identified. Nocturnal bird survey was difficult in the pandemic situation. We used torchlight and sometimes focused it on the branches of trees. We photographed (D7100 DSLR Camera with 200-500 mm VR lens) and identified when any birds were seen. We followed the most authentic Bangladeshi field guide of birds for identification ${ }^{(22,23)}$. Threats to the birds were also noted during fieldwork.

Data analysis: We considered species richness, abundance, relative abundance, observation status, diversity index, and habitat similarity index for calculation. Species richness expresses the number of species, while abundance marks the total number of individuals. The relative abundance of particular bird species was calculated following the formula:

$$
\text { Relative abundance }=\frac{\text { Number of individual of a species }}{\begin{array}{c}
\text { Total number of individuals of all } \\
\text { species }
\end{array}} \times 100
$$

We determined the observation status for each species following $\operatorname{Khan}^{(23)}$. The diversity indices were calculated following the Shannon-Wiener index ${ }^{(24)}$, Simpson's index ${ }^{(25)}$ of diversity. Evenness, which measures the community's equity, was enumerated by dividing the diversity indices with the natural log of species richness. The habitat similarity plot or cluster analysis for microhabitats was performed following the method by the Bray-Curtis index ${ }^{(26)}$. All analyses were done in spreadsheet and PAST software ${ }^{(27)}$.

\section{Results and Discussion}

Species composition: A total of 134 birds with 4338 individuals under 17 orders and 51 families were observed (Table 1). This represents $19.56 \%$ of the total bird species of Bangladesh $^{(17)}$. The number of non-passerine bird species (72 species, $53.37 \%$ ) was more than passerine bird species (62 species, 46.63\%). The highest number of bird species was observed under the order Passeriformes (62 species, 46.63\%), followed by Cuculiformes (10 species, 7.46\%), Coraciiformes (9 species, 6.71\%), and Pelecaniformes (7 species, 5.22\%). The highest number of birds belonged to the family Cuculidae (10 Species, $7.46 \%$ ).

Among 134 species, 115 (85.82\%) were residents and the rest 19 (14.07\%) were migratory birds (Table 1). The migratory bird species Clamator jacobinus, Cuculus micropterus and Merops philippinus were summer migrants and Cuculus canorus was passage migrant. This study was conducted in the summer and rainy season during the lockdown period of the COVID-19 pandemic; hence the number of winter migrant birds was meager (15 species, $11.11 \%)$. There is no published scientific study for comparison about the birds of the study area. 
Table 1. List of avifauna in urban landscape in Jamalpur Sadar Upazila, from March 2020October 2020.

\begin{tabular}{|c|c|c|c|c|c|c|}
\hline Scientific Name & English Name & RA & $\mathrm{MH}$ & ST & $\mathrm{SN}$ & OS \\
\hline Accipiter badius & Shikra & 0.05 & $\mathrm{~T}$ & RS & $\mathrm{R}$ & RE \\
\hline Acridotheres fuscus & Jungle Myna & 3.00 & $\mathrm{RS}, \mathrm{MF}, \mathrm{T}$ & RS & $S, R$ & VC \\
\hline Acridotheres ginginianus & Bank Myna & 1.78 & $\begin{array}{l}\text { GL, FL, RS, } \\
\text { MF }\end{array}$ & RS & $S, R$ & VC \\
\hline Acridotheres tristis & Common Myna & 3.64 & RS, T, FL, GL & RS & $S, R$ & VC \\
\hline Acrocephalus agricola & Paddyfield Warbler & 0.05 & $\mathrm{BU}$ & WV & S & RE \\
\hline Acrocephalus dumetorum & Blyth's Reed-warbler & 0.09 & $\mathrm{BU}$ & WV & S & RE \\
\hline Actitis hypoleucos & Common Sandpiper & 0.23 & MF & RS & S & $\mathrm{UC}$ \\
\hline Aegithina tiphia & Common Iora & 1.01 & $\mathrm{~T}$ & RS & $S, R$ & VC \\
\hline Alcedo atthis & Common Kingfisher & 0.67 & $\mathrm{~T}$ & RS & $\mathrm{S}, \mathrm{R}$ & VC \\
\hline Amaurornis phoenicurus & White-breasted Waterhen & 3.50 & PW, TW, T & RS & $S, R$ & VC \\
\hline Anastomus oscitans & Asian Openbill & 1.27 & $\mathrm{~T}$ & RS & $S, R$ & VC \\
\hline Anthus hodgsoni & Olive-backed Pipit & 0.07 & GL & WV & S & RE \\
\hline Anthus rufulus & Paddyfield Pipit & 0.41 & GL & RS & S & $\mathrm{C}$ \\
\hline Apus nipalensis & House Swift & 2.28 & $\mathrm{US}, \mathrm{T}$ & RS & S & VC \\
\hline Ardea alba & Great White Egret & 0.16 & $\mathrm{~T}$ & RS & $S, R$ & UC \\
\hline Ardea intermedia & Intermediate Egret & 0.74 & PW, TW & RS & $S, R$ & VC \\
\hline Ardeola grayii & Indian Pond Heron & 0.44 & PW, T,GL, TW & RS & $S, R$ & $\mathrm{C}$ \\
\hline Artamus fuscus & Ashy Woodswallow & 0.39 & US,T & RS & $S, R$ & $\mathrm{C}$ \\
\hline Athene brama & Spotted Owlet & 0.44 & $\mathrm{~T}$ & RS & $S, R$ & VC \\
\hline Bubulcus ibis & Cattle Egret & 1.15 & $\begin{array}{l}\text { PW, GL, TW, } \\
\text { FL }\end{array}$ & RS & $S, R$ & VC \\
\hline Cacomantis merulinus & Plaintive Cuckoo & 0.28 & $\mathrm{~T}$ & RS & $\mathrm{S}, \mathrm{R}$ & $\mathrm{C}$ \\
\hline Cacomantis passerinus & Grey-bellied Cuckoo & 0.05 & $\mathrm{~T}$ & RS & S & RE \\
\hline Centropus bengalensis & Lesser Coucal & 0.02 & $\mathrm{~T}$ & RS & S & RE \\
\hline Centropus sinensis & Greater Coucal & 0.02 & GL & RS & $S$ & RE \\
\hline Ceryle rudis & Pied Kingfisher & 0.32 & $\mathrm{~T}$ & RS & $S, R$ & $\mathrm{C}$ \\
\hline Charadrius dubius & Little Ringed Plover & 0.44 & MF & RS & $\mathrm{S}, \mathrm{R}$ & $\mathrm{UC}$ \\
\hline Cisticola juncidis & Zitting Cisticola & 0.32 & GL & RS & $S, R$ & $\mathrm{C}$ \\
\hline Clamator jacobinus & Jacobin Cuckoo & 0.07 & $\mathrm{~T}$ & SV & $S, R$ & UC \\
\hline Columba livia & Rock Dove & 3.25 & US,T & RS & $\mathrm{S}$ & VC \\
\hline
\end{tabular}


Table 1 contd.

\begin{tabular}{|c|c|c|c|c|c|c|}
\hline Scientific Name & English Name & RA & $\mathrm{MH}$ & ST & $\mathrm{SN}$ & OS \\
\hline Copsychus saularis & Oriental Magpie-robin & 2.97 & $\mathrm{RS}, \mathrm{T}, \mathrm{FL}$ & RS & $S, R$ & VC \\
\hline Coracias affinis & Indochinese Roller & 0.37 & $\mathrm{~T}$ & RS & $S, R$ & $\mathrm{C}$ \\
\hline Coracias benghalensis & Indian Roller & 0.67 & $\mathrm{~T}$ & RS & $S, R$ & $\mathrm{C}$ \\
\hline Coracina macei & Large Cuckoos-shrike & 0.05 & $\mathrm{~T}$ & RS & $\mathrm{R}$ & $\mathrm{RE}$ \\
\hline Coracina melanoptera & $\begin{array}{l}\text { Black-headed Cuckoo- } \\
\text { shrike }\end{array}$ & 0.05 & $\mathrm{~T}$ & RS & S & $\mathrm{RE}$ \\
\hline Coracina melaschistos & $\begin{array}{l}\text { Black-winged Cuckoo- } \\
\text { shrike }\end{array}$ & 0.05 & $\mathrm{~T}$ & WV & S & $\mathrm{RE}$ \\
\hline Corvus levaillantii & Jungle Crow & 1.29 & $\mathrm{RS}, \mathrm{T}$ & RS & $S, R$ & VC \\
\hline Corvus splendens & House Crow & 2.90 & US, T, RS & RS & $S, R$ & VC \\
\hline Cuculus canorus & Common Cuckoo & 0.02 & $\mathrm{~T}$ & PV & S & $\mathrm{RE}$ \\
\hline Cuculus micropterus & Indian Cuckoo & 0.14 & $\mathrm{~T}$ & SV & S & $\mathrm{UC}$ \\
\hline Cypsiurus balasiensis & Asian Palm Swift & 1.41 & $\mathrm{~T}, \mathrm{US}$ & RS & $S, R$ & VC \\
\hline Dendrocitta vagabunda & Rufous Treepie & 0.41 & $\mathrm{~T}$ & RS & $S, R$ & VC \\
\hline Dendrocopos macei & $\begin{array}{l}\text { Fulvous-breasted } \\
\text { Woodpecker }\end{array}$ & 1.13 & $\mathrm{~T}$ & RS & $S, R$ & VC \\
\hline Dendrocygna javanica & Lesser Whistling Duck & 1.27 & PW & RS & $S, R$ & VC \\
\hline Dicaeum erythrorhynchos & Pale-billed Flower pecker & 0.39 & $\mathrm{~T}$ & RS & $S, R$ & VC \\
\hline Dicrurus aeneus & Bronzed Drongo & 0.02 & $\mathrm{~T}$ & RS & S & $\mathrm{RE}$ \\
\hline Dicrurus leucophaeus & Ashy Drongo & 0.02 & $\mathrm{~T}$ & WV & S & $\mathrm{RE}$ \\
\hline Dicrurus macrocercus & Black Drongo & 1.80 & $\mathrm{~T}$, US & RS & $S, R$ & VC \\
\hline Dinopium benghalense & Black-rumped Flameback & 1.06 & $\mathrm{~T}$ & RS & $S, R$ & $\mathrm{VC}$ \\
\hline Egretta garzetta & Little Egret & 1.50 & GL, TW, MF & RS & $S, R$ & $\mathrm{VC}$ \\
\hline Elanus caeruleus & Black-winged Kite & 0.23 & $\mathrm{PW}, \mathrm{T}$ & RS & $S, R$ & $\mathrm{UC}$ \\
\hline Eudynamys scolopaceus & Western Koel & 1.52 & $\mathrm{~T}$ & RS & $S, R$ & VC \\
\hline Falco chicquera & Red-headed Falcon & 0.02 & $\mathrm{~T}$ & RS & $\mathrm{R}$ & RE \\
\hline Falco tinnunculus & Common Kestrel & 0.05 & $\mathrm{~T}$ & WV & S & RE \\
\hline Ficedula albicilla & Taiga Flycatcher & 0.07 & $\mathrm{~T}$ & WV & S & RE \\
\hline Gallinula chloropus & Common Moorhen & 0.05 & PW & RS & S & RE \\
\hline Halcyon smyrnensis & White-breasted kingfisher & 0.95 & $\mathrm{~T}$ & RS & $S, R$ & $\mathrm{VC}$ \\
\hline Haliastur indus & Brahminy Kite & 3.11 & $\mathrm{~T}, \mathrm{US}$ & RS & $S, R$ & VC \\
\hline Hierococcyx varius & Common Hawk-Cuckoo & 0.18 & $\mathrm{~T}$ & RS & $S, R$ & $\mathrm{C}$ \\
\hline
\end{tabular}


Table 1 contd.

\begin{tabular}{|c|c|c|c|c|c|c|}
\hline Scientific Name & English Name & RA & $\mathrm{MH}$ & ST & SN & OS \\
\hline Hirundo rustica & Barn Swallow & 0.37 & $\mathrm{~T}$ & RS & $S, R$ & C \\
\hline Hypothymis azurea & Black-naped Monarch & 0.25 & $\mathrm{~T}$ & RS & $S, R$ & $\mathrm{UC}$ \\
\hline Ixobrychus cinnamomeus & Cinnamon Bittern & 0.02 & TW & RS & $\mathrm{R}$ & RE \\
\hline Ixobrychus sinensis & Yellow Bittern & 0.05 & PW & RS & $\mathrm{R}$ & $\mathrm{UC}$ \\
\hline Ketupa zeylonensis & Brown Fish Owl & 0.02 & $\mathrm{~T}$ & RS & $S$ & RE \\
\hline Lanius cristatus & Brown Shrike & 0.12 & $\mathrm{~T}, \mathrm{BU}$ & $\mathrm{WV}$ & $S$ & $\mathrm{UC}$ \\
\hline Lanius schach & Long-tailed Shrike & 0.67 & $\mathrm{~T}, \mathrm{US}$ & RS & $\mathrm{S}, \mathrm{R}$ & VC \\
\hline Lanius tephronotus & Grey-backed Shrike & 0.02 & $\mathrm{~T}$ & RS & $\mathrm{S}$ & RE \\
\hline Larus brunnicephalus & Brown-headed Gull & 0.16 & PW & RS & S & RE \\
\hline Lonchura malabarica & White-throated Munia & 0.39 & GL, T & RS & $S, R$ & C \\
\hline Lonchura malacca & Tricoloured Munia & 0.25 & $\mathrm{~T}, \mathrm{GL}$ & RS & S & $\mathrm{UC}$ \\
\hline Lonchura punctulata & Scaly-breasted Munia & 3.02 & GL & RS & $S, R$ & VC \\
\hline Lonchura striata & White-rumped Munia & 0.53 & $\mathrm{FL}, \mathrm{GL}$ & RS & $S, R$ & RE \\
\hline Malacocincla abbotti & Abbott's Babbler & 0.05 & $\mathrm{~T}$ & RS & S & C \\
\hline Megalurus palustris & Striated Grassbird & 0.14 & GL & RS & $S, R$ & $\mathrm{UC}$ \\
\hline Merops leschenaulti & $\begin{array}{l}\text { Chestnut-headed Bee- } \\
\text { eater }\end{array}$ & 0.05 & $\mathrm{~T}$ & RS & S & RE \\
\hline Merops orientalis & Asian Green Bee-eater & 0.71 & $\mathrm{~T}$ & RS & $S, R$ & C \\
\hline Merops philippinus & Blue-tailed Bee-eater & 0.44 & $\mathrm{~T}$ & SV & $S$ & $\mathrm{UC}$ \\
\hline Metopidius indicus & Bronze-winged Jacana & 1.08 & PW & RS & $S, R$ & C \\
\hline Microcarbo niger & Little Cormorant & 1.45 & TW, PW & RS & $S, R$ & $\mathrm{VC}$ \\
\hline Celeus brachyurus & Rufous Woodpecker & 0.05 & $\mathrm{~T}$ & RS & $\mathrm{R}$ & RE \\
\hline Milvus migrans & Black Kite & 1.89 & US & RS & $S, R$ & $\mathrm{VC}$ \\
\hline Mirafra assamica & Rufous-winged Lark & 0.07 & $\mathrm{~T}$ & RS & S & RE \\
\hline Motacilla alba & White Wagtail & 0.07 & $\mathrm{MF}$ & WV & S & $\mathrm{RE}$ \\
\hline Motacilla madaraspatensis & White-browed Wagtail & 0.25 & $\mathrm{MF}$ & RS & $S, R$ & $\mathrm{UC}$ \\
\hline Nectarinia asiatica & Purple Sunbird & 0.92 & $\mathrm{~T}$ & RS & $S, R$ & VC \\
\hline Nectarinia zeylonica & Purple-rumped Sunbird & 0.46 & $\mathrm{~T}$ & RS & $S, R$ & $\mathrm{VC}$ \\
\hline Ninox scutulata & Brown Boobook & 0.35 & $\mathrm{~T}$ & RS & $S, R$ & $\mathrm{VC}$ \\
\hline Nisaetus cirrhatus & Changeable Hawk-eagle & 0.02 & $\mathrm{~T}$ & RS & $\mathrm{R}$ & RE \\
\hline Oriolus xanthornus & Black-hooded Oriole & 1.27 & $\mathrm{~T}$ & RS & $S, R$ & VC \\
\hline
\end{tabular}


Table 1 contd.

\begin{tabular}{|c|c|c|c|c|c|c|}
\hline Scientific Name & English Name & RA & $\mathrm{MH}$ & ST & $\mathrm{SN}$ & OS \\
\hline Orthotomus sutorius & Common Tailorbird & 1.29 & BU, FL, RS & RS & $S, R$ & $\mathrm{VC}$ \\
\hline Otus lettia & Collared Scops Owl & 0.02 & $\mathrm{~T}$ & RS & $\mathrm{R}$ & $\mathrm{RE}$ \\
\hline Parus major & Great Tit & 0.60 & $\mathrm{~T}$ & RS & $S, R$ & VC \\
\hline Passer domesticus & House Sparrow & 1.43 & $\mathrm{~T}, \mathrm{US}, \mathrm{BU}$ & RS & $S, R$ & VC \\
\hline Passer montanus & Eurasian Tree Sparrow & 2.72 & US, T, RS & RS & $S, R$ & VC \\
\hline Pelargopsis capensis & Stork-billed Kingfisher & 0.23 & $\mathrm{~T}$ & RS & $S, R$ & UC \\
\hline Pericrocotus cinnamomeus & Small Minivet & 0.58 & $\mathrm{~T}$ & RS & S & UC \\
\hline Pernis ptilorhyncus & Oriental Honey Buzzard & 0.16 & $\mathrm{~T}$ & RS & $S, R$ & UC \\
\hline Phaenicophaeus tristis & Green-billed Malkoha & 0.12 & $\mathrm{~T}$ & RS & $\mathrm{R}$ & UC \\
\hline Phylloscopus fuscatus & Dusky Warbler & 0.09 & BU & WV & S & RE \\
\hline Phylloscopus inornatus & Inornate Warbler & 0.05 & BU & WV & $S$ & RE \\
\hline Phylloscopus trochiloides & Greenish Warbler & 0.05 & $\mathrm{BU}$ & WV & S & RE \\
\hline Picus xanthopygaeus & $\begin{array}{l}\text { Streak-throated } \\
\text { Woodpecker }\end{array}$ & 0.48 & $\mathrm{~T}$ & RS & $S, R$ & VC \\
\hline Ploceus benghalensis & Black-breasted Weaver & 0.07 & GL & RS & $\mathrm{R}$ & $\mathrm{RE}$ \\
\hline Ploceus philippinus & Baya Weaver & 1.18 & $\mathrm{~T}, \mathrm{GL}$ & RS & $S, R$ & VC \\
\hline Prinia gracilis & Graceful Prinia & 0.51 & $\mathrm{~T}, \mathrm{BU}, \mathrm{GL}$ & RS & $S, R$ & $\mathrm{C}$ \\
\hline Prinia hodgsonii & Grey-breasted Prinia & 0.07 & $\mathrm{BU}$ & RS & S & $\mathrm{RE}$ \\
\hline Prinia inornata & Plain Prinia & 0.18 & GL, BU & RS & $S, R$ & $\mathrm{UC}$ \\
\hline Psilopogon asiaticus & Blue-throated Barbet & 1.61 & $\mathrm{~T}$ & RS & $S, R$ & VC \\
\hline Psilopogon haemacephalus & Coppersmith Barbet & 1.68 & $\mathrm{~T}$ & RS & $S, R$ & VC \\
\hline Psilopogon lineatus & Lineated Barbet & 0.60 & $\mathrm{~T}$ & RS & $S, R$ & VC \\
\hline Psittacula eupatria & Alexandrine Parakeet & 0.28 & $\mathrm{~T}$ & RS & $S, R$ & $\mathrm{C}$ \\
\hline Psittacula krameri & Rose-ringed Parakeet & 2.47 & $\mathrm{~T}$ & RS & $S, R$ & VC \\
\hline Pycnonotus cafer & Red-vented Bulbul & 6.11 & $\mathrm{~T}$ & RS & $S, R$ & VC \\
\hline Rhipidura albicollis & White-throated Fantail & 0.23 & $\mathrm{BU}, \mathrm{T}$ & RS & $S, R$ & $\mathrm{C}$ \\
\hline Rostratula benghalensis & Greater Painted Snipe & 0.25 & TW & RS & $\mathrm{R}$ & $\mathrm{UC}$ \\
\hline Spilopelia chinensis & Eastern Spotted Dove & 1.57 & US, T, AG & RS & $S, R$ & VC \\
\hline Spilornis cheela & Crested Serpent Eagle & 0.05 & $\mathrm{~T}$ & RS & S & RE \\
\hline Streptopelia decaocto & Eurasian Collared Dove & 0.16 & GL, FL, US & RS & $S, R$ & UC \\
\hline Streptopelia tranquebarica & Red Turtle Dove & 0.92 & AG, GL & RS & $S, R$ & VC \\
\hline
\end{tabular}


Table 1 contd.

\begin{tabular}{|c|c|c|c|c|c|c|}
\hline Scientific Name & English Name & RA & $\mathrm{MH}$ & ST & $\mathrm{SN}$ & OS \\
\hline Sturnus contra & Asian Pied Starling & 3.62 & FL, RS, T, US & RS & $S, R$ & VC \\
\hline Sturnus malabaricus & Chestnut-tailed Starling & 2.60 & $\mathrm{~T}$ & RS & $S, R$ & $\mathrm{VC}$ \\
\hline $\begin{array}{l}\text { Tephrodornis } \\
\text { pondicerianusz }\end{array}$ & Common Woodshrike & 0.09 & $\mathrm{~T}$ & RS & $S$ & RE \\
\hline Terpsiphone paradisi & Asian Paradise Flycatcher & 0.18 & $\mathrm{~T}$ & RS & $S, R$ & $\mathrm{UC}$ \\
\hline $\begin{array}{l}\text { Threskiornis } \\
\text { melanocephalus }\end{array}$ & Black-headed Ibis & 0.05 & TW & WV & $\mathrm{R}$ & $\mathrm{RE}$ \\
\hline Treron phoenicopterus & $\begin{array}{l}\text { Yellow Footed Green } \\
\text { Pigeon }\end{array}$ & 0.05 & $\mathrm{~T}$ & RS & S & $\mathrm{RE}$ \\
\hline Tringa glareola & Wood Sandpiper & 0.07 & MF & WV & $S$ & $\mathrm{RE}$ \\
\hline Tringa ochropus & Green Sandpiper & 0.09 & MF & WV & $S$ & $\mathrm{RE}$ \\
\hline Turdoides earlei & Striated Babbler & 0.23 & GL & RS & $\mathrm{R}$ & $\mathrm{RE}$ \\
\hline Turdoides striata & Jungle Babbler & 0.39 & $\mathrm{~T}, \mathrm{BU}$ & RS & $\mathrm{S}, \mathrm{R}$ & $\mathrm{UC}$ \\
\hline Tyto alba & Common Barn Owl & 0.02 & $\mathrm{~T}$ & RS & $S$ & $\mathrm{RE}$ \\
\hline Upupa epops & Common Hoopoe & 0.74 & FL,GL, T & RS & $S, R$ & $\mathrm{C}$ \\
\hline Vanellus cinereus & Grey-headed Lapwing & 0.07 & $\mathrm{MF}$ & RS & S & $\mathrm{RE}$ \\
\hline Vanellus indicus & Red-wattled Lapwing & 0.83 & GL, MF & RS & $\mathrm{S}, \mathrm{R}$ & $\mathrm{C}$ \\
\hline Zoothera citrina & Orange-headed Thrush & 0.09 & $\mathrm{BU}$ & RS & $S, R$ & $\mathrm{UC}$ \\
\hline Zosterops palpebrosus & Oriental White-eye & 0.14 & $\mathrm{~T}$ & RS & $S$ & RE \\
\hline
\end{tabular}

(Note: RA- Relative Abundance; MH- Microhabitats, BU- Bushy Area, FL- Fallow Land, RSRoadside, MF- Mudflat, GL- Grassland, T- Tree, US- Urban Settlement, PW- Permanent Waterbody, TW- Temporary Waterbody, AG- Agricultural Land; ST- Status, RsS- Resident, WVWinter Visitor, SV- Summer Visitor; SN-Season, W-Winter, S- Summer and R- Rainy Season; OSObservation Status, VC- Very Common, C-Common, UC- Uncommon, RE- Rare).

Primarily, the Bangladesh Bureau of Statistics enlisted 59 species of birds in 2011 in the Jamalpur district. Compared to other studies on birds in different districts ${ }^{(28-30)}$ in Bangladesh, this study showed a higher number of species except for the Magura Sadar upazila ${ }^{(16)}$. The high species richness in the study area indicates that the urban landscape favored more species due to limited movements of people during the pandemic situation. Although there are no published scientific data on birds from the study area, we assume that the pandemic situation created more opportunities in the free movement of birds with fewer human disturbances.

Richness, abundance and diversity in months and seasons: Species richness and abundance were more in March (89 species, 659 individuals) followed by April (61 species). The lowest individual was counted for in July (499 individuals) (Table 2). In the summer season, we counted 
$52.51 \%(\mathrm{n}=2278)$ of total individuals presenting 120 species higher than rainy season 2060 individuals (47.49\%). The richness and abundance in the two seasons differed but the variation of seasonal diversity was minor. Diversity was the highest in June $\left(H=4.122, D_{s}=0.980\right)$, though the difference was not high considering Simpson's index (Table 2). The calculated evenness was the highest for October (0.788), signifying a higher similarity in this month. At the beginning of summer, some winter migratory birds were observed along with summer migratory birds. Besides, this season is also the breeding period of different resident species sustaining more diversity. In Bangladesh, from March to June movement and general office activities of humans were completely prohibited due to COVID 19, so birds may have more opportunities to move in the urban area ${ }^{(10-12)}$. Therefore, it is assumed that species richness and diversity in March and June have increased due to decreased human movement activity.

Table 2. Species richness (S), abundance (A), evenness (E), diversity [Shannon-Weiner Index (H), Simpson's Index $\left.\left(D_{s}\right)\right]$ in different months, seasons and habitats.

\begin{tabular}{|c|c|c|c|c|c|c|}
\hline \multicolumn{2}{|c|}{ Category/Parameters } & $\mathrm{S}$ & A & $\mathrm{H}$ & $\mathrm{E}$ & $\mathrm{D}_{\mathrm{s}}$ \\
\hline \multirow{8}{*}{ Months } & March & 89 & 659 & 4.087 & 0.669 & 0.978 \\
\hline & April & 61 & 566 & 3.819 & 0.747 & 0.973 \\
\hline & May & 63 & 541 & 3.855 & 0.750 & 0.974 \\
\hline & June & 84 & 512 & 4.122 & 0.734 & 0.980 \\
\hline & July & 75 & 499 & 3.977 & 0.711 & 0.975 \\
\hline & August & 67 & 513 & 3.949 & 0.774 & 0.976 \\
\hline & September & 76 & 520 & 4.083 & 0.780 & 0.980 \\
\hline & October & 64 & 528 & 3.920 & 0.788 & 0.976 \\
\hline \multirow{2}{*}{ Seasons } & Rainy & 95 & 2060 & 4.103 & 0.637 & 0.979 \\
\hline & Summer & 120 & 2278 & 4.168 & 0.534 & 0.978 \\
\hline \multirow{10}{*}{ Micro habitats } & $\mathrm{AG}$ & 2 & 62 & 0.680 & 0.987 & 0.487 \\
\hline & $\mathrm{BU}$ & 14 & 79 & 2.224 & 0.660 & 0.834 \\
\hline & FL & 9 & 131 & 1.938 & 0.771 & 0.828 \\
\hline & GL & 23 & 368 & 2.499 & 0.529 & 0.847 \\
\hline & MF & 11 & 180 & 1.999 & 0.671 & 0.826 \\
\hline & PW & 11 & 187 & 1.866 & 0.588 & 0.800 \\
\hline & RS & 9 & 303 & 1.981 & 0.806 & 0.843 \\
\hline & $\mathrm{T}$ & 94 & 2476 & 3.876 & 0.513 & 0.969 \\
\hline & TW & 9 & 157 & 1.806 & 0.677 & 0.807 \\
\hline & US & 14 & 395 & 2.294 & 0.708 & 0.879 \\
\hline \multirow{3}{*}{ Macro habitats } & Aquatic & 24 & 546 & 2.714 & 0.629 & 0.921 \\
\hline & Arboreal & 94 & 2502 & 3.882 & 0.516 & 0.969 \\
\hline & Terrestrial & 51 & 1290 & 3.323 & 0.544 & 0.950 \\
\hline
\end{tabular}


Richness, abundance and diversity in habitats: We observed mostly arboreal species (94 species, $\mathrm{n}=2502$ ) followed by terrestrial ( 51 species, $\mathrm{n}=1290$ ) and aquatic ( 24 species, $\mathrm{n}=546$ ) species. Among the observed birds, 94 species representing $57.67 \%$ of total individuals were found in trees which were the highest. The diversity indices also showed the uppermost diversity $\left(\mathrm{H}=3.876, \mathrm{D}_{\mathrm{s}}=0.969\right)$ in the tree among all types of microhabitats. All birds (including terrestrial and aquatic) use the tree for nesting, roosting, and resting sites, although their niche usage can differ. This turns the tree into a diverse source of microhabitat. The richness of native trees species is very high in our study areas, especially in residential, office areas, homestead forests, roadside areas which supported more species to get shelter, food, roosting and nesting. The evenness was the highest $(\mathrm{E}=0.987)$ in the agricultural land area in the study area (Table 2). Agricultural land is usually similar in type and vegetation structure; thus, it can harbor birds with the same food preference. This also elucidates the higher evenness of aquatic bird species than other macrohabitats (Table 2).

To determine the similarity among 10 types of microhabitat, we performed the Bray-Curtis similarity index. Three large clusters were formed in this index. Roadside area and fallow land formed a cluster, where permanent water bodies and temporary water bodies formed the second and tree and urban settlement formed the third cluster. Roadside area and fallow lands show more similarities with mudflats and formed another cluster. Then grassland formed another cluster with the roadside area, fallow lands and mudflats. Later, another large cluster was formed among roadside areas, fallow lands, mudflats, and grasslands with tree and urban settlement. This plot also showed that agricultural land and bush formed individual clusters among all microhabitats (Fig. 2).

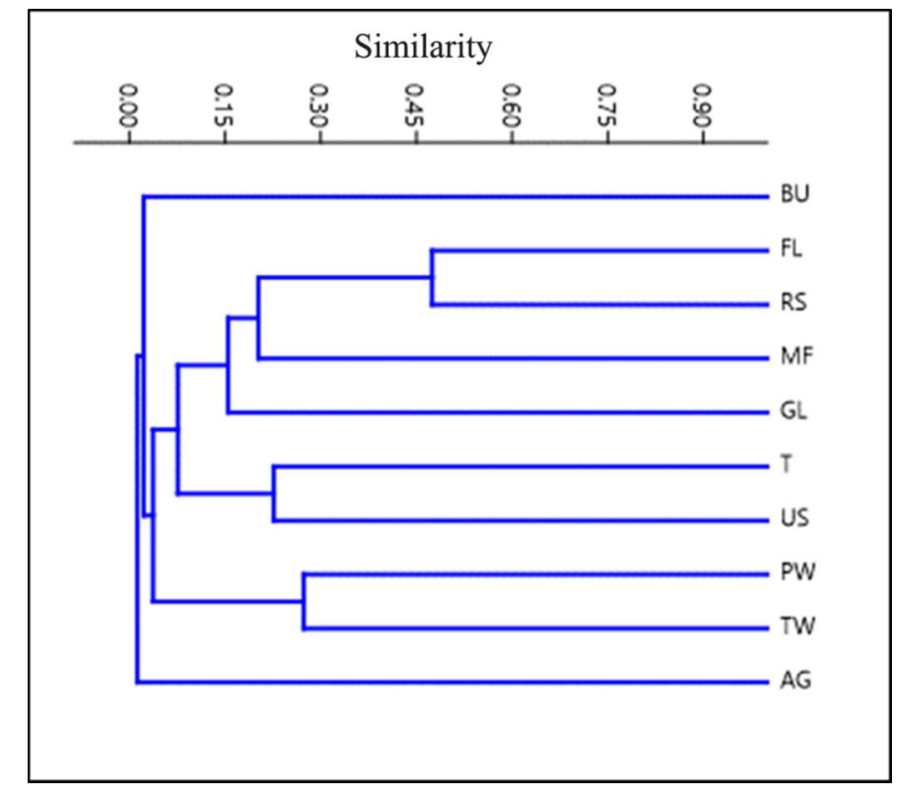

Fig. 2. Similarity profile test among microhabitats using Bray-Curtis index. 
Relative abundance and observation status: Red-vented Bulbul Pycnonotus cafér was the most abundant bird species with the highest relative abundance $(6.11 \%)$. Observation status showed that 49 (36.29\%) birds were very common, 18 (33.33\%) were common, 24 (17.77\%) were uncommon and 44 (32.59\%) were rare. Pycnonotus cafer mainly feeds on fruits, grain, nectar, and insects ${ }^{(17)}$. Covid-19 lockdown stops worsening habitat and allows birds to visit the study ground more frequently than past. Besides, the breeding season of this species starts in March and continues to August, which might have caused the higher detectability and count of the species.

Observed threats and threatened status: Among the observed bird species, all birds are categorized as least concern (LC) according to IUCN Red List Bangladesh (2015) except Threskiornis melanocephalus, which is categorized as vulnerable (VU). During the study period, major anthropogenic activities and urbanization processes were not observed due to the Covid-19 pandemic. Different bird species (e.g., Metopidius indicus, Anastomus oscitans, Amaurornis phoenicurus, Streptopelia tranquebarica, Lonchura malacca, Lonchura punctulata, Passer montanus, Ploceus philippinus, Threskiornis melanocephalus, Picus xanthopygaeus and Dendrocygna javanica) moved very frequently around the human habitat in the urban Llandscape of the study area. But, plastic pollution was repeatedly observed, and people were reluctant to dispose of surgical instruments like mask and gloves inappropriate places.

In conclusion, this study shows the scenario of avifaunal diversity in the urban area of Jamalpur Sadar upazila during lockdown due to the COVID-19 pandemic. A detailed study is essential for understanding the wildlife and ecological services of them in this area, which will help take proper management and conservation plan for avifauna. Awareness creation among local people, especially young communities, is essential in conserving and protecting habitats.

\section{Acknowledgement}

We are very much grateful to Mr. Md. Mazharul Islam for his kind support for preparing the study area map.

\section{References}

1. Rocha EA and MDE Fellowes 2018. Does urbanization explain differences in interactions between an insect herbivore and its natural enemies and mutualists? Urban Ecosyst. 21:405-417.

2. McKinney ML 2006. Urbanization as a Major Cause of Biotic Homogenization. Biol. Conserv.127: 247-60.

3. Bolwig S, D Pomeroy, H Tushabe and D Mushabe. 2006. Crops, trees, and birds: Biodiversity change under agricultural intensification in Uganda's farmed landscapes. GeografiskTidsskrift-Danish. J. Geogr. 106(2): 115-130.

4. Evans KL, DE Chamberlain, BJ Hatchwell, RD Gregory and KJ Gaston 2011. What makes an urban bird? Glob. Change Biol. 17(1): 32-44.

5. Strohbach MW, D Haaseand and N Kabisch 2009. Birds and the city: urban biodiversity, land use, and socioeconomics. Ecol. Soc. 14(2): 31.

6. Gatesire T, D Nsabimana, ANyiramana, JL Seburanga and MO Mirville 2014. Bird diversity and distribution in relation to urban landscape types in Northern Rwanda. Sci. World J. 2014: 1-12. 
7. Jessop TS, P Smissen, F Scheelings and T Dempster 2012. Demographic and phenotypic effects of human mediated trophic subsidy on a large Australian lizard (Varanus varius): meal ticket or last supper?. PLoS one 7(4): e34069.

8. Rebolo-Ifrán N, JL Tella and M Carrete 2017. Urban conservation hotspots: predation release allows the grassland-specialist burrowing owl to perform better in the city. Sci. Rep. 7(1): 1-9.

9. Gibbs JP, MF Buff and BJ Cosentino 2019. In: Hall M and S Balogh (Eds), Understanding Urban Ecology, The Biological System-Urban Wildlife, Adaptation, and Evolution: Urbanization as a Driver of Contemporary Evolution in Gray Squirrels (Sciurus carolinensis). Springer, Cham, pp. 269-286.

10. Sahagun L 2020. Coyotes, falcons, deer and other wildlife are reclaiming L.A. territory as humans stay at home. Los Angeles Times. Available at: https://www. latimes.com/environment/story/2020-0421/wild life-thrives-amid-coronavirus-lockdown

11. Gordo O, L Brotons, S Herrando and G Gargallo 2021. Rapid behavioural response of urban birds to COVID-19 lockdown. Proc. R. Soc. B. 288(1946): 20202513.

12. Zellmer AJ, EM Wood, T Surasinghe, BJ Putman, GB Pauly, SB Magle, SL Jesse, AMK Cria and M Fidino 2020. What can we learn from wildlife sightings during the COVID-19 global shutdown? Ecosphere 11(8): e03215.

13. Venter ZS, K Aunan, S Chowdhury and J Lelieveld 2020. COVID-19 lockdowns cause global air pollution declines. Proc. Natl Acad. Sci. 117: 18984- 18990.

14. Lecocq T, SP Hicks, K van Noten, K van Wijk, P Koelemeijer, RSM de Plaen, F Massin, G Hillers, RE Anthony, MT Apoloner and H Xiao 2020. Global quieting of high-frequency seismic noise due to COVID-19 pandemic lockdown measures. Science 369(6509): 1338-1343.

15. IUCN Bangladesh 2015. Red List of Bangladesh Vol. 3. IUCN, International Union for Conservation of Nature, Bangladesh Country Office, Dhaka, Bangladesh. pp xvi+676.

16. Shome AR, MM Alam, MF Rabbe, MM Rahman and MF Jaman 2020. Diversity, status and habitat usage of avifauna at sadar upazila, Magura, Bangladesh. Bangladesh J. Zool. 48(2): 441-456.

17. Khan MMH 2018. A Photographic Guide to Wildlife of Bangladesh. Arannayk Foundation, Dhaka, Bangladesh. pp. 488.

18. Jaman MF, SU Sarker and NJ Sarker 1999. Food habits and feeding behavior of black drongo, Dicrurus macrocercus albirictus (Hodgson). Bangladesh J. Zool. 26(2): 57-66.

19. Rajia S, MM Alam, GW Chowdhury, M Akash and MA Islam 2015. Status and diversity of birds of Ramna Park, Dhaka, Bangladesh. Bangladesh J. Zool. 43(2): 291-301.

20. Islam N, MF Jaman, MM Rahman and MM Alam. 2018. Wildlife Diversity and Population Status of Kashimpur Union, Gazipur, Bangladesh. J. Asiat. Soc. Bangladesh, Sci. 44(2): 101-115.

21. Gaston AJ 1975. Methods for estimating bird populations. J. Bomb. Nat. Hist. Soc. 72: 271-273.

22. Khan MAR 2015. Wildlife of Bangladesh-checklist and guide. Chayabithi, Purana Paltan, Dhaka 1000, pp. 568.

23. Halder RR 2010. A photographic guide to the bird of Bangladesh. Baikal Teal Publication, Dhaka, Bangladesh, pp. 257.

24. Shannon CE and $\mathrm{W}$ Wiener 1949. The mathematical theory. University of Illinois press, Urbana, pp. 117.

25. Simpson EH 1949. Measurement of diversity. Nature 163: 688.

26. Bray JR and JT Curtis 1957. An ordination of the upland forest communities of southern Wisconsin. Ecol. Monogr. 27(4): 325-349.

27. Hammer $\varnothing$, DAT Harper and PD Ryan 2001. PAST: Palaeontological Statistics software package for education and data analysis. Palaeontol. Electron. 4:1-9. 
28. Jaman MF, MS Rahman and ME Haque 2011. Diversity of avifauna at the Bangladesh Academy for Rural Development (BARD), Kotbari, Comilla. Univ. J. Zool. Rajshahi Univ. 30: 41-44.

29. Karmakar S, S Parween and AS Reza 2011. Birds of Joypurhat district, Bangladesh. J. Life Earth Sci. 6: 51-57.

30. Amin A and KA Hasan 2019. Checklist and relative diversity of avifauna of Atrai, Raninagar and Naogan Sadar upazillas of Naogaon district of Bangladesh. Int. J. Zoo. Studies. 4(6): 38-45.

(Manuscript received: 26 April 2021; accepted: 30 May, 2021) 